\title{
Gambling, motor cautiousness, and choice impulsivity: An experimental study
}

\author{
ANDREW HARRIS*, DARIA KUSS and MARK D. GRIFFITHS \\ International Gaming Research Unit, Psychology Division, Nottingham Trent University, Nottingham, UK \\ (Received: March 28, 2018; revised manuscript received: August 1, 2018; second revised manuscript received: September 12, 2018; \\ accepted: September 19, 2018)
}

\begin{abstract}
Background: Impulsivity is currently more commonly regarded as multifaceted, comprising both motor and cognitive subdomains. However, it is less clear how distinct these subdomains are, and the extent to which they interact and draw upon the same psychological resources. Methods: The present experiment comprised 70 regular (non-problem) gamblers, and investigated the potential to induce impulsivity transfer effects within an electronic gambling context. Original and existing harm-minimization approaches were tested for their efficacy in inducing motor cautiousness during an electronic slot machine simulation. Participants were exposed to a forced discriminatory motor choice procedure, or pop-up responsible gambling messages that either contained emotive or non-emotive responsible gambling content. The subsequent impact these interventions had on delay discounting and reflection impulsivity was also measured using the 27-item Monetary Choice Questionnaire and Information Sampling Task, respectively. Results: Findings demonstrated that only original harm-minimization approaches, which force the gambler to engage in discriminatory motor choice procedures during gambling, were successful in inducing motor cautiousness. However, both the discriminatory choice procedure and emotive message harm-minimization approaches were successful in facilitating cognitive choice, even though the emotive message intervention was unsuccessful in facilitating motor response inhibition, suggesting both an indirect motor cautiousness route, and a more direct route to improved cognitive choice during gambling. Conclusion: This study demonstrated that decision-making during gambling can be improved by making simple structural changes to slot machine platforms, by encouraging active engagement in motor processes, which result in a transfer of cautiousness to wider cognitive domains.
\end{abstract}

Keywords: executive control, gambling, impulsivity, harm minimization, motor cautiousness

\section{INTRODUCTION}

Impulsivity has been defined as behavior characterized by little or no forethought, reflection, or consideration of the consequences (van den Bos, 2007). Several research studies have noted that the construct of impulsivity should be considered multifaceted, comprising subdomains representing distinct processes (for a review, see Arce \& Santisteban, 2006). A number of scholars (e.g., Bechara, 2002; Bechara, Damasio, \& Damasio, 2000) make the distinction between motor impulsivity and cognitive/choice impulsivity. Motor impulsivity, which refers to action without thinking (Kocka \& Gagnon, 2014), is considered the antithesis of motor response inhibition, typically assessed using the Go/No-Go Task, although it can also be assessed with the Continuous Performance Test (e.g., Holmes et al., 2002) and the Stop Signal Task (e.g., Ávila, Cuenca, Félix, Parcet, \& Miranda, 2004). Motor impulsivity is usually associated with disruption to the dorsolateral prefrontal cortex (Bechara et al., 2000). For example, individuals with frontal cortex lesions are more likely to display risky and more impulsive behavior when it is contextually inappropriate (Duncan, 1986; Shallice, 1982). Brain stimulation studies utilizing techniques, such as transcranial magnetic stimulation (TMS), show that similar patterns of behavior are found in healthy individuals when these brain regions are stimulated (e.g., Chambers et al., 2006; Verbruggen, Aron, Stevens, \& Chambers, 2010).

Cognitive impulsivity is considered the inability to weigh the consequences of immediate and future events, with higher levels of cognitive impulsivity being associated with an inability to delay gratification (Arce \& Santisteban, 2006). Cognitive impulsivity is typically assessed with approaches including the Iowa Gambling Task (Bechara, Damasio, Damasio, \& Anderson, 1994), as well as experimental delay discounting tasks (Matta, Gonçalves, \& Bizarro, 2012), including the Monetary Choice Questionnaire (MCQ; Kirby, Petry, \& Bickel, 1999). Although still associated with prefrontal cortex regions in the brain, lesion studies suggest that the ventromedial region of the prefrontal cortex is the main area involved in cognitive impulsivity (Bechara, 2002).

More recent research into human impulsivity has resulted in an increased understanding of the neurobiological mechanisms involved in impulsivity, leading to the view that impulsivity is not a unitary construct. However, while

\footnotetext{
* Corresponding author: Andrew Harris; International Gaming Research Unit, Psychology Division, Nottingham Trent University, 50 Shakespeare Street, Nottingham NG1 4GQ, UK; Phone: +44 115 84 88434; E-mail: Andrew.harris2015@my.ntu.ac.uk
}

This is an open-access article distributed under the terms of the Creative Commons Attribution-NonCommercial 4.0 International License, which permits unrestricted use, distribution, and reproduction in any medium for non-commercial purposes, provided the original author and source are credited, a link to the CC License is provided, and changes - if any - are indicated. 
psychopathological research has identified distinctions between impulsive choice and action in disorders including attention-deficit hyperactivity disorder (ADHD; Solanto et al., 2001) and substance abuse (Broos, Diergaarde, Schoffelmeer, Pattij, \& De Vries, 2012; Diergaarde et al., 2008), there is also evidence suggesting a considerable overlap between neurotransmitter systems and brain regions involved in impulsive choice and impulsive action (Chambers, Garavan, \& Bellgrove, 2009; Peters \& Buchel, 2011). This brings into debate the degree to which these two constructs are distinct. The aims of this study were therefore to assess the nature of this relationship between impulsive choice and action within a gambling context, with a view to assessing the impact of gambling harm-minimization tools in reducing levels of impulsivity displayed by gamblers within a gambling session.

\section{Evidence for distinction and overlaps between impulsive choice and impulsive action}

Broos, Schmaal, et al. (2012) examined the relationship between impulsive choice and impulsive action among 101 healthy university student participants. They compared within-participant delay discounting (impulsive choice) with performance on a modified continuous performance task (immediate and delayed memory task) and a Stop Signal Task (impulsive action). Performance on these behavioral tasks was also compared with self-report impulsivity using the Barratt Impulsiveness Scale (BIS-11; Patton, Stanford, \& Barratt, 1995). Results showed that the immediate and delayed memory task performance correlated positively with each other, but there was no correlation between performance on these tasks assessing impulsive action with delay discounting rates in the delayed discounting task assessing impulsive choice. In addition, there was only a weak correlation between the delayed memory task and Stop Signal Task performance. Furthermore, BIS-11 scores failed to correlate significantly with any of the behavioral measures of impulsivity. Their results suggest that impulsive choice and impulsive action are largely unrelated in healthy adults.

However, contemporary neuroscience research offers an alternative account of the relationship between impulsive action and impulsive choice. For example, Knoch et al. (2006) used TMS to demonstrate that when the dorsolateral prefrontal cortex - an area associated with the suppression of impulsive motor actions (Bogacz, Wagenmakers, Forstmann, $\&$ Nieuwenhuis, 2010) - is disrupted from normal functioning, it leads to increased risk-taking within a gambling context. Such results have led to arguments that controlling impulses across a variety of domains including suppression of thoughts, decisions, and actions, relies on overlapping inhibitory control networks. However, direct evidence is rare because the empirical findings are typically correlational in nature (Verbruggen, Adams, \& Chambers, 2012).

Studies have directly compared the neural components active in a range of tasks assessing self-control and the suppression of impulsive choice and action (for a review, see Cohen \& Lieberman, 2010). Such studies typically compare motor response inhibition performance with other forms of self-control, utilizing measures such as the Flanker
Task, which requires the suppression of distracting information (Bunge, Dudukovic, Thomason, Vaidya, \& Gabrieli, 2002; Wager et al., 2005), and the Wisconsin Card Sorting Test, which assesses set-shifting and perseverance (Konishi et al., 1999). Combined neuroimaging techniques typically show that the right inferior frontal cortex and/or right anterior insula are active during inhibition trials within such tasks, regions that are associated with response inhibition in experiments utilizing Go/No-Go and Stop Signal paradigms (Bunge et al., 2002; Wager et al., 2005). Such studies indicate that various forms of self-control - a construct viewed as the antithesis of impulsivity (Bickel et al., 2012) share underlying neural components.

Muraven and Baumesiter (2000) conducted a series of studies requiring participants to complete tasks involving various aspects of self-control, including the suppression of impulsive motor actions in a Stop Signal Task, as well as tasks requiring suppression of thoughts, emotions, and temptation. Participants who were asked to perform a second impulse control task generally performed worse on that task than participants who were initially asked to complete an effort-matched task not requiring self-control. These results are explained using a shared resources account of self-control, suggesting that self-control is not only a unified process, but that various aspects of self-control are derived from a limited resource that is prone to fatigue effects.

\section{Impulsivity transfer effects within a gambling context}

Within a gambling context, Verbruggen et al. (2012) demonstrated that participants encouraged to exercise response inhibition and monitor their motor actions demonstrated a preference for less risky gambling choices in a novel gambling task. Requiring participants to exercise a degree of motor cautiousness in the presence of potential stop signals resulted in participants making gambling-related decisions that were less risky. In addition, participants spent more time making decisions as indicated by longer choice latencies. This line of research was later extended by Stevens et al. (2015) who examined whether the relationship between motor cautiousness and monetary decisions was driven by changes in arousal and/or perceptual processing style. The results showed that inducing motor cautiousness with the presence of stop signals resulted in participants selecting smaller and less risky betting choices, but that the relationship was not mediated by the stop signals adjusting the perceptual processing pattern assessed using eye tracker technology, nor was the effect mediated by the stop signals adjusting participant's arousal levels, as assessed using skin conductance responses. One explanation for these transfer phenomena offered by Stevens et al. (2015) is that the presence of stop signals adjusted the hedonic and motivational value of subsequent gambling-related stimuli, and is consistent with Dickinson and Dearing's (1979) suggestion of an antagonistic appetite and aversive center within the brain. One key limitation of these findings is that they were based on a novel gambling procedure that does not replicate a typical gambling product structure. In addition, the procedure that is used to assess the choice component of impulsivity, while having real-world application, is not a 
recognized and standardized test of impulsive choice, making the findings difficult to compare with wider research assessing the relationship between different aspects of impulsivity.

The potential for impulsivity transfer effects within a gambling context provides the opportunity for harmminimization approaches that are designed to facilitate motor response inhibition to also influence a gambler's wider decision-making capacity within a gambling session. Although research has provided competing views regarding the relationship between subcomponents of impulsivity, neurobiological and behavioral evidence suggests that there are both distinct and shared mechanisms involved within different aspects of impulsivity (Chambers et al., 2009).

\section{Relevance to the present study}

If a link exists between impulsive action and impulsive choice, then it may be possible to positively influence the choice component of impulsivity by inducing a more cautious motor response style. From a gambling harmminimization perspective, it is important that any proposed approach be tested for unintentional perverse effects. For example, evidence suggests that different aspects of selfcontrol rely on a shared pool of resources (Muraven \& Baumesiter, 2000), meaning that the application of motor control during gambling could negatively affect performance on choice impulsivity tasks via fatigue effects.

\section{Research aims and hypotheses}

The first aim of the experiment was to experimentally assess if inducing motor cautiousness during a slot machine gambling simulation would result in a cautiousness transfer effect to performance on choice impulsivity tasks. The range of aforementioned evidence from neuroscience and behavioral studies demonstrates significant overlaps between impulsive action and impulsive choice, both within and outside of a gambling context. This led to the hypothesis that inducing motor cautiousness during slot machine gambling will result in less impulsive performances on subsequent choice impulsivity tasks (H1).

If inducing a less impulsive and more controlled motor response style within a gambling context has an impact on choice impulsivity, it should also intuitively follow that inducing a rapid and highly impulsive motor response style could also influence decision-making style on choice impulsivity tasks. The second research aim of the experiment was therefore to examine the impact of inducing a rapid motor response style during slot machine gambling on subsequent performance on impulsive choice tasks. It was hypothesized that inducing a rapid motor response style during a slot machine gambling simulation would result in greater levels of impulsivity being demonstrated in subsequent impulsive choice tasks (H2).

The third aim of the experiment was to assess the efficacy of new and existing harm-minimization tools in facilitating more cautious decision-making during a gambling session. It was hypothesized that harm-minimization tools successful in reducing impulsive actions would lead to reduced levels of impulsive choice (H3).

\section{METHODS}

\section{Design}

A between-participants experiment was conducted to assess the impact of new and existing gambling harm-minimization tools on impulsivity transfer effects. An electronic slot machine simulator was designed using a combination of the graphical user interface and coding function available on the Psychopy experiment builder (Peirce, 2007; Figure 1). Behavioral measures of response inhibition were built into the slot machine simulator, and following each session of gambling, participants were given various online psychometric scales to complete to assess subjective arousal, dissociation, valence, and perceived self-control. All scales were presented and completed using the Psychopy experiment builder. Reaction time was also measured, which is a standard function in the experimental software. Choice impulsivity tasks were then conducted using the same computer platform, in the form of a 27-item MCQ (Kirby et al., 1999) and Information Sampling Task (IST; Clark, Robbins, Ersche, \& Sahakian, 2006; Parke, Harris, Parke, \& Goddard, 2015).

\section{Participants}

A sample of 70 ( $n=53$ males $)$ non-problem, regular gamblers were recruited from amusement arcades and sports clubs in the Lincolnshire areas of the UK. These areas were targeted during the recruitment process as they were identified as areas likely to contain a high density of gamblers. All participants were classed as regular gamblers, defined for the purposes of this study as an individual who had gambled at least once per month in the past 12 months. Participant's mean age was 31.14 years $(S D=10.90)$, with ages ranging from 18 to 60 years. A short screening questionnaire was administered to ensure that participants: (a) reported regular participation in gambling and (b) had never suffered from a gambling problem or were currently suffering from a gambling problem. An affirmative answer on either count of

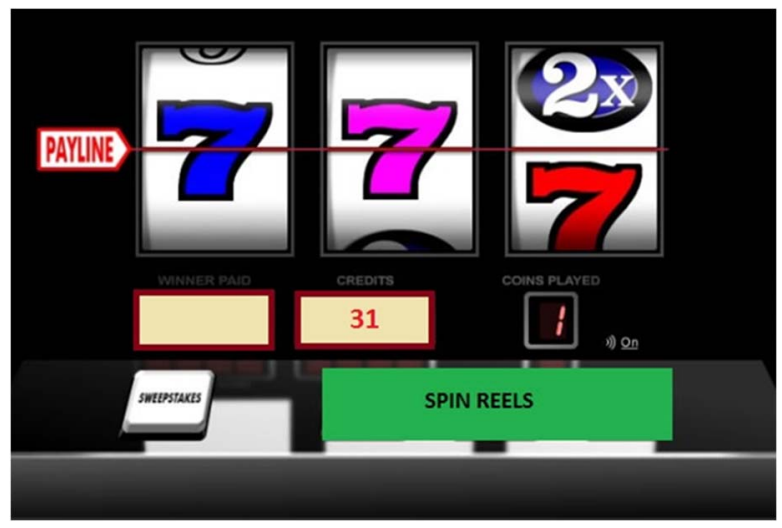

Figure 1. EGM simulator screenshot. A three-reeled slot machine simulator with a single pay line was designed using the Psychopy experiment builder. The machine is activated using the space bar on the participant's keyboard when the visual display spin button changes from gray to either green or red (although participants are instructed to withhold responses when the button is red) 
problem gambling resulted in participants being excluded from participation. Consequently, five participants were excluded from participation following the screening questionnaire, as they were found to have previously experienced problem gambling.

\section{Materials}

Behavioral Response Inhibition Task. The electronic game machine (EGM) simulator consisted of 90 trials (gambling events) per condition. The EGM was activated by pressing the "spin button," which was the space bar on a standard computer keyboard. The spin button on the EGM simulator's visual display varied in color from green to red, with green trials indicating participants could spin the reels and continue gambling, but red indicating that they need to withhold their motor response. Response inhibition was therefore measured with an "online" behavioral Go/No-Go Task, because the task was embedded into the EGM simulator. The first 30 trials of each condition were all green "go" responses, often referred to as a "training phase" in response inhibition tasks (e.g., Verbruggen et al., 2012). The purpose of the first 30 trials all being "go" trials was to allow any prepotent patterns of motor responses to develop. The remaining 60 trials in each condition consisted of a randomized 4:1 ratio of green "go" and red "no-go" trials.

The Go/No-Go Task. This task is considered to be the gold-standard measure of motor inhibition (Arce \& Santisteban, 2006). In comparison to other experimental paradigms that measure motor inhibition, the Go/No-Go Task is simple to administer and understand, and it can be used with varying types of stimuli, including both verbal and nonverbal variants, as well as providing multiple behavioral data to assist the examination of processes that may be involved in response inhibition (e.g., reaction time). Moreover, the Go/No-Go Task has been widely used as a measure of motor impulsivity in a wide variety of domains and settings within the field of psychology (e.g., Langley et al., 2004; Matthews, Simmons, Arce, \& Paulus, 2005; Rosello, Munar, Justo, \& Arias, 1998; Spinella, 2004).

Jacobs' 4-item Measure of Dissociation. Dissociation in a gambling context can be characterized as absorption in the game where thoughts of personal well-being are laid to one side and gamblers seem no longer aware of other events going on around them (Delfabbro, 2006). One of the potential uses for pop-up responsible gambling messages, such as those tested within the present experiment, is to interrupt these dissociative states that can be experienced by gamblers. Therefore, dissociation represents a psychological construct that may shed light on the relationship between responsible gambling tools, motor impulsivity, and choice impulsivity. Dissociative experience was measured in this study using a modified version of Jacobs' 4-item Measure of Dissociation (Kuley \& Jacobs, 1988). The original scale was modified in two ways for this study. First, the original four items were modified to ask participants to reflect on the gambling session they had just participated in, as opposed to gambling experience in general. For example, the question "When gambling, how often do you feel like you have been in a trance?" was modified to read "Thinking back to the gambling session you have just completed, how often did you feel like you were in a trance?" The second modification of the scale was the addition of a fifth item, asking participants about their perception of time during the gambling session, an item incorporated into previous experimental gambling research (Blaszczynski, Cowley, Athony, \& Kinsley, 2016; Gupta \& Derevensky, 1998). All five items were self-reports on a 5-point Likert scale, anchored at 1 ("never") and 5 ("all the time"). Midpoint of the scale ("3") indicated "occasionally."

Self-assessment manikin (SAM). Participant's subjective levels of arousal and valence during each experimental condition were assessed using the SAM (Lang, 1980). The SAM is a non-verbal pictorial assessment technique that directly assesses the pleasure and arousal associated with an individual's affective reaction to a wide variety of stimuli (Figure 2). The SAM was chosen to assess valence and arousal, because it is a method that has been demonstrated as an easy way to administer, non-verbal method for quickly assessing the arousal, and pleasure associated with an individual's reaction to an event or stimuli. SAM scores measuring experience of arousal are highly correlated with scores obtained using verbal and lengthier semantic differential scales (Bradly \& Lang, 1994). They have also been used to assess emotional responses to a wide range of stimuli, including both pictures (e.g., Lang, Greenwald, Bradly, \& Hamm, 1993) and sounds (e.g., Bradly, 1994), and successfully administered among a range of clinical populations, as well as children and non-English speakers (Bradley \& Lang, 1994). Full body versions of the SAMs were used for both the valence and arousal scale (portraitonly versions are available for the valence scale, in which

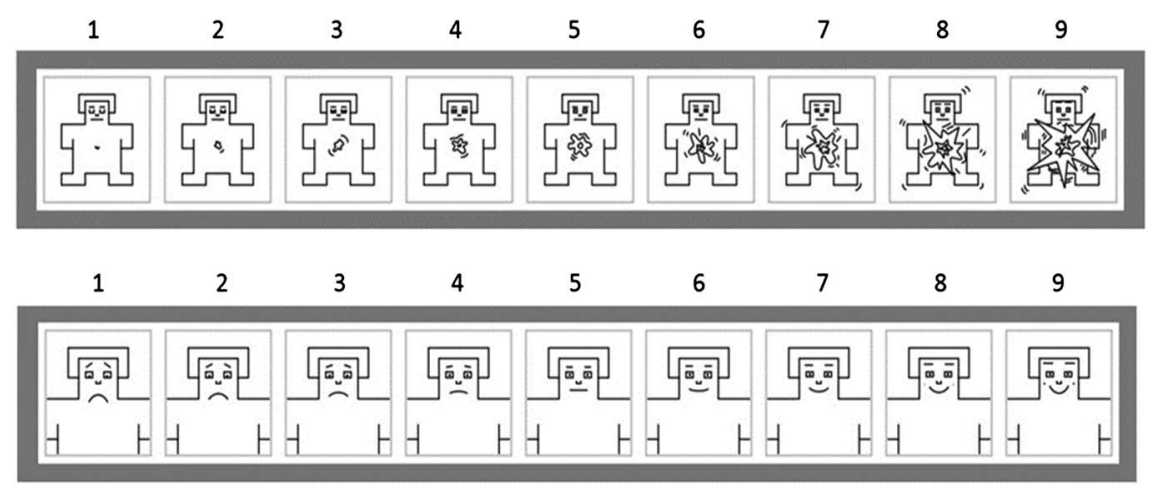

Figure 2. Arousal (top) and valence (bottom) of self-assessment manikins 
only a close up of the manikin face is presented), and both scales were presented in their 9-point scale versions (Figure 2).

\section{Choice impulsivity measures}

Delay discounting. The 27-item MCQ is a delay discounting task where participants have to choose between a smaller immediate hypothetical monetary reward, or a larger but temporally delayed reward. The questionnaire varies in both the amount of hypothetical money on offer, as well as the hypothetical temporal delay between the immediate and delayed reward. Each question was presented individually on a computer screen. The left-hand side of the screen always read "Would you prefer £x today" and the right-hand screen always read "or $£ x$ in $x$ days," where the delayed reward was always higher in value. Participants made their choices by pressing the corresponding number next to the two options, " 1 " for the immediate reward and " 0 " for the delayed reward. These numbers were spatially related to the options on screen. This was also done to minimize erroneous key presses, because they appeared on opposite ends of the number scale on the keyboard. An overall preference for immediate reward over larger but delayed rewards has been reliably shown to indicate higher levels of choice impulsivity (Anokhin, Golosheykin, \& Mulligan, 2015).

Reflection impulsivity. The IST is a measure of reflection impulsivity. The IST in the present experiment was a modified version of the IST developed by Clark et al. (2006). The original IST was a grid of $5 \times 5$ covered boxes that contained one of two unseen colors, and participants were required to sequentially select a box to reveal the color underneath the cover. Participants were required to identify which of the two colors would be in the majority when all boxes were uncovered.

In this study, the IST was modified to be more representative of a familiar probability task, and was based upon the classic "Urn Problem" (see Parke et al., 2015 for use of this task in a gambling context). The urn contained 19 unseen balls, and each ball was colored black or red. Participants were required to determine whether red or black balls within the urn were in the majority. Participants were able to gather information by selecting to remove a ball from the urn to reveal its color. Points were awarded in the IST for correct predictions regarding which color was in the majority. For each IST trial, participants began with 95 points, and had five points removed for each ball taken from the urn. For example, if a participant removed three balls from the urn, and correctly predicted that red was in the majority they would win 80 points for that IST trial. However, if participants made an incorrect prediction, then they would be fined 100 points for that trial. Participants were informed that all of the points that they accumulated over the IST trials would be totaled, and the top five participants who accumulated the most points would receive a $£ 50$ shopping voucher as a prize.

During the experiment, participants were not provided with feedback regarding whether their predictions in each IST trial were correct. This is also a modification on classic ISTs, because feedback on correct/incorrect responses is normally provided following each trial. Because this study wanted to control for valence as a covariate, it was decided that no feedback would be given following each trial to prevent emotional carryover contamination effects (i.e., to prevent the results from one trial changing the emotional state of the participant in later trials which may have the undesirable effect of facilitating or impeding performance). As a result of this control, any differences in performance across conditions can be more confidently attributed to the experimental manipulations.

Reflection impulsivity was assessed by observing three component variables: Mean Information Sampled, Mean Response Latency, and Mean Probability of Making Correct Decisions ( $p$-correct). Mean Information Sampled referred to the mean average number of balls removed from the urn for each IST trial, and the Mean Response Latency refers to the mean amount of time taken to make a decision to remove another ball or to make a prediction of which color was in the majority. Mean Probability of Making Correct Decisions ( $p$-correct) referred the probability of the participants' color predictions being accurate, based on the available information at the time the decision was made (Clark et al., 2006; Parke et al., 2015).

\section{Procedure}

Each participant was randomly assigned to gamble on a three-reeled EGM simulator in one of five conditions: no intervention (control condition), emotive pop-up message intervention, informative pop-up message intervention, structure change intervention, and a double-response condition. Participants were provided with $£ 9$ to gamble with (10p per spin) and were told that any money they had left at the end of the experiment could be kept. The event frequency of the slot machine simulator was $1.5 \mathrm{~s}$, because this speed has been shown to be the most problematic for exercising response inhibition in a previous experiment by the present authors (names removed for blind review). Each slot machine condition was programmed to give the illusion of randomness. However, the slot machines were preprogrammed to control for volume, frequency, and range of wins, as well as number of near misses (Clark, Lawrence, Astley-Jones, \& Gray, 2009). The slot machine pay back percentage was $90 \%$, although participants were allowed to keep the full $£ 9$ as a compensation for their time, but they were not informed of this until the end of the experiment.

Participants were given a tutorial on how to operate the EGM simulator, and were informed of what each of the visual display features was, including the pay-line, credit balance, and win totals on winning spins. A pay-out structure was also shown to participants during the tutorial, showing how much money would be won for specific matching symbols (Figure 3). Participants were instructed to only operate the machine by pressing the spin button (space bar on standard computer keyboard) when the spin button on the visual display was green in color, and instructed they must withhold from pressing the spin button when it was red in color. The slot machine was programmed to spin automatically on "no-go" trials after a delay equivalent to one event frequency $(1.5 \mathrm{~s})$. The first 30 trials of each slot machine condition were all "go" trials, and the remaining 60 trials consisted of a $4: 1$ ratio of "go" to "no-go" trials. 


\begin{tabular}{|c|c|}
\hline 2 & 100 \\
\hline 7 & 50 \\
\hline & 30 \\
\hline & 25 \\
\hline 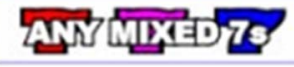 & 20 \\
\hline 场 场 & 15 \\
\hline BAR EAR EAR & 10 \\
\hline ANY ANY ANY & 5 \\
\hline ANT OWI 2x & 2 \\
\hline
\end{tabular}

Figure 3. EGM simulator pay-out structure. Participants were provided with the pay-out structure information during the tutorial. Each number is a multiple of one credit, with one credit being equal to $10 \mathrm{p}$. Therefore, prizes for winning symbols ranged from $20 \mathrm{p}$ to $£ 10$

\section{Structure change condition}

With the exception of the structure change condition and double-response condition, the EGM simulator was operated by pressing the space bar on a standard keyboard when the spin button was green in color on the visual display. However, in the structure change condition, the operation of the EGM simulator consisted of pressing either the left arrow key or the right arrow key, depending on the image of the black directional arrow displayed within the green spin button on the visual display. During the double-response condition, participants were instructed to press the spin button (i.e., space bar) twice in rapid succession to operate the machine, inducing a rapid response style.

\section{Pop-up message intervention}

In both pop-up message intervention conditions, a pop-up message appeared on screen for $30 \mathrm{~s}$ after the 30th trial. The message could not be skipped, and gambling could only continue once the message had been on screen for $30 \mathrm{~s}$. Depending upon the condition, the message either displayed general information about potential dangers relating to gambling, or displayed emotional content related to potential familial and financial consequences related to reckless gambling (Figure 4). Following each gambling condition, participants were asked to complete the arousal SAM, valence SAM, Dissociative Experience Scale, and singleitem measure of perceived self-control, in that order. All scales were completed online immediately following the gambling simulation in each condition.

The choice impulsivity tasks followed the completion of the various scales, separated by a message that appeared on screen for $5 \mathrm{~s}$ informing participants that the cognitive choice tasks were about to start. All five IST trials were presented first, each with a different combination of preprogrammed to-be-removed red and black balls, and finally, participants completed the 27-item MCQ online.

\section{Ethics}

Before commencement of the study, the study was approved by the research team's University Ethics Committee. The study protocol was designed in accordance with guidelines of the Declaration of Helsinki. Participants were fully briefed and instructed on how to complete both tasks prior to the beginning of the experiment and provided their informed consent to participate in the study. Participants were informed that all the data were confidential and anonymous.

\section{RESULTS}

\section{BIS-11 and age controls}

Mean participant trait impulsivity scores were assessed using the BIS-11 (Patton et al., 1995). A betweenparticipants analysis of variance (ANOVA) showed that participants' overall BIS-11 scores $[F(4,69)=0.149$, $p=.963$ ], including the second-order factors motor impulsivity $[F(4,69)=0.801, p=.529]$, attentional impulsivity $[F(4,69)=0.924, p=.455]$, and non-planning impulsivity $[F(4,69)=0.141, p=.966]$, did not differ between groups to a statistically significant degree. In addition, a betweenparticipants ANOVA showed that participants' mean ages did not differ to a statistically significant degree between experimental groups $[F(4,69)=0.348, p=.844]$.

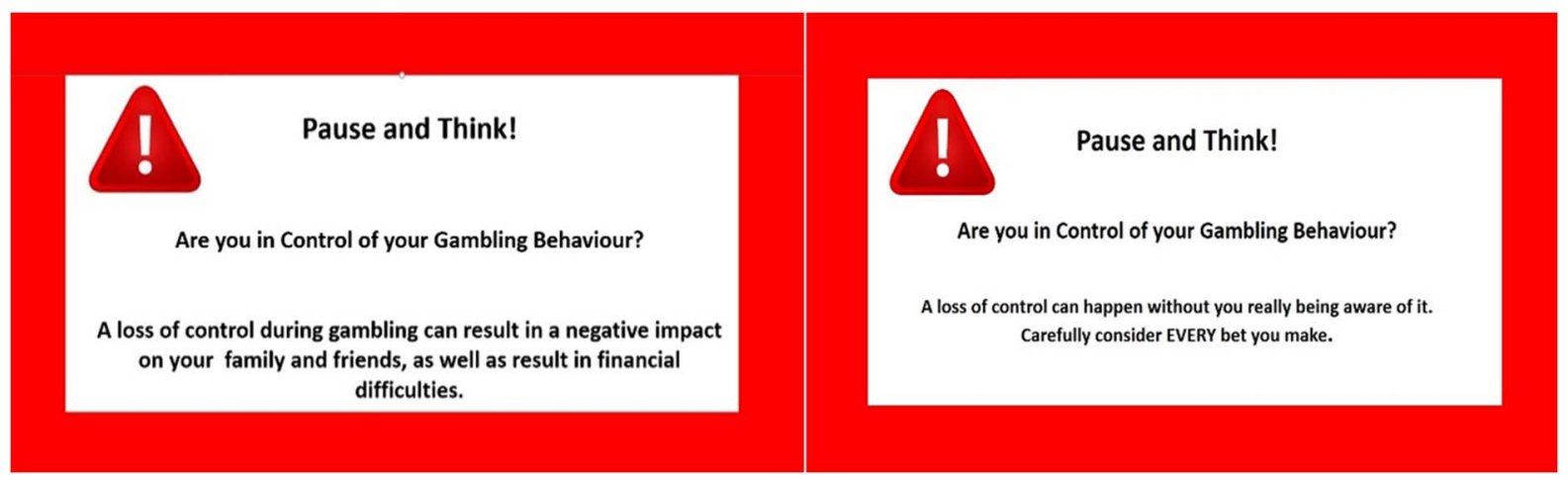

Figure 4. Emotive pop-up message (left) and informative message (right). Pop-up message was presented after 30 gambling trials, interrupting play for $30 \mathrm{~s}$ before allowing the gambling simulation to continue 
Therefore, differences in the dependent variables assessed at the group level throughout the experiment can be more confidently attributed to the experimental manipulations, as opposed to participant preexisting levels of trait impulsivity and/or age effects. All means and standard deviations for the BIS-11 and age values are shown in Table 1.

\section{Arousal}

A one-way between-participants ANOVA showed a non-statistically significant effect of intervention on mean participant reported levels of arousal $[F(4,69)=0.867$, $\left.p=.489, \eta^{2}=0.05\right]$. The results also showed that mean arousal ratings in all conditions, which were conducted at fast gambling speeds, were moderately high. Mean arousal ratings in all conditions were above six on the 9-point scale, with a grand mean of $6.39(S D=0.98)$, confirming the subjectively arousing experiencing of the gambling simulation conducted at a high event frequency (Table 2).

\section{Dissociation}

A one-way between-participants ANOVA showed that mean levels of dissociation experienced by the participants did not vary to a statistically significant level between groups $\left[F(4,69)=0.336, p=.852, \eta^{2}=0.02\right]$. Overall, dissociation levels in all conditions while gambling at fast speeds of play were very low, with grand mean dissociation levels $(7.00, S D=1.53)$ falling close to the bottom end of the possible range of scores (5-25). All dissociation means and standard deviations are reported in Table 2.
Valence

A one-way between-participants ANOVA showed that difference in mean valence ratings between conditions did not vary to a statistically significant degree $[F(4,69)=0.257$, $\left.p=.904, \eta^{2}=0.02\right]$. In all conditions, mean valence ratings were above the midpoint of the 9-point scale (grand mean = $5.79, S D=1.07)$, indicating that on average, participants found the gambling experience overall moderately pleasurable, and that none of the harm-minimizations interventions had deleterious effects on the emotional experience during the gambling simulation. All valence means and standard deviations are reported in Table 2.

\section{Reaction time}

A one-way between-participants ANOVA showed that the difference in mean reaction times between conditions was statistically significant $\left[F(4,69)=13.09, p<.001, \eta^{2}=\right.$ $0.45]$. The fastest mean reaction time was found in the double-response condition $(0.56 \mathrm{~s}, S D=0.09)$. Bonferroni pairwise comparisons showed that this mean reaction time was statistically significantly faster when compared to the control condition $(0.72 \mathrm{~s}, S D=0.12, p=.01, d=1.51)$, emotive message condition ( $0.71 \mathrm{~s}, S D=0.13 ; p=.022$, $d=1.34)$, informative message condition $(0.70 \mathrm{~s}, S D=$ $0.13, p=.031, d=1.25)$, and structure change condition $(0.90 \mathrm{~s}, S D=0.14, p<.001, d=2.89)$. The slowest mean reaction time was found in the structure change condition, which was statistically significantly slower than the control condition $(p=.004, d=1.38)$, emotive message condition

Table 1. Participant's means (and standard deviations) of age and BIS-11 scores across experimental conditions

\begin{tabular}{lccccc}
\hline & \multicolumn{5}{c}{ Variable } \\
\cline { 2 - 6 } Condition & BIS-11 non-planning & BIS-11 motor & BIS-11 attention & BIS-11 overall & Age \\
\hline Control (CONT) & $21.57(3.34)$ & $21.28(3.20)$ & $16.79(2.15)$ & $59.64(7.02)$ & $33.79(13.25)$ \\
Structure change (STRU) & $21.35(3.00)$ & $21.21(2.78)$ & $16.86(1.83)$ & $59.43(5.32)$ & $30.71(9.83)$ \\
Emotive message (EMOT) & $20.92(2.84)$ & $20.21(2.78)$ & $16.78(2.08)$ & $57.93(6.39)$ & $29.07(11.62)$ \\
Informative message (INFO) & $21.14(3.59)$ & $20.50(3.65)$ & $17.21(2.72)$ & $58.86(8.65)$ & $30.43(10.40)$ \\
Double-response (DOUB) & $21.64(21.64)$ & $22.07(2.73)$ & $15.57(3.13)$ & $59.29(4.77)$ & $31.71(10.13)$ \\
\hline
\end{tabular}

Note. No statistical significance was found between-groups for all variables. BIS-11: Barratt Impulsiveness Scale.

Table 2. Means (and standard deviations) of arousal, valence, dissociation, reaction time, and response inhibition scores across experimental conditions

\begin{tabular}{lccccc}
\hline & \multicolumn{4}{c}{ Dependent variable } \\
\cline { 2 - 5 } Condition & $\begin{array}{c}\text { Arousal } \\
(1-9)\end{array}$ & $\begin{array}{c}\text { Valence } \\
(1-9)\end{array}$ & $\begin{array}{c}\text { Dissociation } \\
(5-25)\end{array}$ & Reaction time (s) & $\begin{array}{c}\text { Response inhibition } \\
(\% \text { successfully inhibited) }\end{array}$ \\
\hline Control & $6.29(1.33)$ & $5.57(1.16)$ & $7.29(1.77)$ & $0.72(0.12)$ & $66.67(12.23)$ \\
Structure change & $6.43(0.51)$ & $5.50(1.09)$ & $6.71(1.38)$ & $0.90(0.14)$ & $83.93(10.57)$ \\
Emotive message & $6.14(0.95)$ & $5.42(1.02)$ & $7.21(1.72)$ & $0.71(0.13)$ & $69.05(11.05)$ \\
Informative message & $6.29(1.07)$ & $5.71(1.20)$ & $6.86(1.41)$ & $0.70(0.13)$ & $67.86(14.57)$ \\
Double-response & $6.79(0.89)$ & $5.79(0.97)$ & $6.93(1.49)$ & $0.56(0.09)$ & $57.14(12.17)$ \\
\hline
\end{tabular}

Note. Only differences in mean reaction time and response inhibition scores reached statistical significance $(p<.001)$. Statistically significant Bonferroni comparisons: Reaction Time - DOUB < CONT, STRU, EMOT, INFO. STRU > CONT, EMOT, INFO, DOUB. Response Inhibition - STRU > CONT, EMOT, INFO, DOUB. CONT: control; STRU: structure change; EMOT: emotive message; INFO: informative message; DOUB: double-response. 
$(p=.002, d=1.41)$, informative message condition $(p=.001$, $d=1.48)$, and the double-response condition $(p<.001$, $d=2.89$ ). There was neither statistically significant difference between mean reaction time in the emotive message and control conditions, the informative message and control conditions, nor between the emotive and informative message conditions. Therefore, the results indicate that only the structure change condition was successful in slowing participant's mean reaction times when compared to a control group, whereas inducing a rapid response style with the double-response condition sped up participant's mean reaction time.

\section{Response inhibition performance}

A one-way between-participants ANOVA showed that the difference in mean response inhibition performance between conditions was statistically significant $[F(4,69)=8.71$, $\left.p<.001, \eta^{2}=0.35\right]$. Performance was highest in the structure change condition $(83.93 \%, S D=10.57)$. Bonferroni pairwise comparisons showed that this mean response inhibition performance in the structure change condition was statistically significantly higher when compared to the control condition $(66.67 \%, S D=12.23, p=.004, d=1.51)$, emotive message condition $(69.05 \%, \quad S D=11.05, \quad p=.02$, $d=1.38)$, informative message condition $(67.86 \%$, $S D=14.57, p=.009, d=1.26)$, and double-response condition $(57.14 \%, S D=12.17, p<.001, d=2.35)$. While performance was lowest in the double-response condition, pairwise comparisons failed to show this mean performance was statistically significantly worse than the control, emotive message, or the informative message conditions. All other pairwise comparisons showed non-statistically significant results. On the whole, only the structure change condition facilitated response inhibition performance relative to a control condition, and worked significantly better when compared to the emotive and informative message interventions. Inducing a rapid response style using the double-response condition resulted in the worst response inhibition performance overall, but this failed to reach statistical significance.

\section{Information Sampling Task}

p-correct. The main variable of interest regarding reflection impulsivity, as measured by the IST, was the probability of a participant being correct at the time of making a decision, which is referred to as $p$-correct. $p$-correct was calculated using the following formula (Clark et al., 2006; Parke et al., 2015):

$$
\mathrm{P}(\text { Correct })=\frac{\sum_{\mathrm{k}-\mathrm{A}}^{\mathrm{z}}\left(\begin{array}{l}
\mathrm{z} \\
\mathrm{k}
\end{array}\right)}{2^{\mathrm{z}}},
$$

where $z=19-$ (number of balls removed from the urn), and $\mathrm{A}=13$ - (number of balls removed of selected color).

A one-way between-participants ANOVA showed a statistically significant difference between mean $p$-correct scores across conditions $\left[F(4,69)=7.41, p<.001, \eta^{2}=0.31\right]$. Bonferroni pairwise comparisons showed that participants in the emotive message condition $(84.49 \%, S D=3.71$, $p=.024, d=0.61)$ and structure change condition
$(85.93 \%, S D=2.67, p=.008, d=1.22)$, had statistically significantly higher $p$-correct values when compared to the control group $(82.40 \%, S D=3.11)$. However, there was no statistically significant difference between mean $p$-correct scores in the emotive message and structure change conditions. The $p$-correct values for the informative message $(81.88 \%, S D=2.91)$ and double-response $(80.72 \%, S D=$ 4.21 ) conditions did not differ significantly when compared to a control group. Performance was significantly worse in the double-response condition when compared to the emotive message $(p=.003, d=0.95)$ and structure change $(p=.001, d=1.47)$ conditions. Performance was also significantly worse in the informative message condition when compared to the structure change condition $(p=.02$, $d=1.45$ ), but the difference in mean $p$-correct values between the informative message and emotive message conditions just failed to reach statistical significance at the $p<.05$ level $(p=.054, d=0.78)$. Therefore, the results indicate that the use of a structure change intervention and emotive message intervention is able to facilitate probabilistic decision-making during gambling, when compared to a control group. The use of a structure change intervention also facilitated performance above that of an informative message intervention, while the trend in the data suggest emotive messaging also facilitated performance above that of an informative message intervention, and the $p$ value just failed to reach statistical significance.

\section{Response latency}

The mean time that took participants to arrive at a decision in the IST was compared across conditions using a one-way between-participants ANOVA. Results showed that the difference in means was statistically significant $[F(4,69)=7.37$, $\left.p<.001, \quad \eta^{2}=0.31\right]$ Bonferroni pairwise comparisons showed that only the mean time taken in the structure change (9.19 s, $S D=2.78, p=.027, d=1.20)$ and emotive message conditions (9.76 s, $S D=3.22, p=.005, d=1.30)$ differed from the control condition $(5.93 \mathrm{~s}, S D=2.65)$ to a statistically significant level, where participants took significantly more time to make a decision. Significantly less time was taken to make a decision in the double-response condition $(5.19 \mathrm{~s}$, $S D=2.28)$ compared to the emotive message condition $(p<.001, d=1.64)$ and the structure change condition $(p=.003, d=1.57)$. All other pairwise comparisons failed to reach statistical significance at the $p<.05$ level.

\section{Information sampled}

Mean information sampled refers to the mean number of balls removed (i.e., sampled) from the urn during the IST, before a decision on predicted majority color was made. The results from the one-way between-participants ANOVA showed a significant difference between the mean amount of information sampled across conditions $[F(4,69)=7.40$, $\left.p<.001, \eta^{2}=0.31\right]$. Bonferroni pairwise comparisons showed that when compared to a control group (3.51 s, $S D=1.21$ ), only the mean amount of information sampled in the structure change condition $(5.14 \mathrm{~s}, S D=1.28)$ differed to a statistically significant level $(p=.012, d=1.31)$, where participants on average sampled more information 
before making their decision. Significantly more information was sampled in the structure change condition when compared to the informative message $(3.51 \mathrm{~s}, S D=1.32$; $p=.012, d=1.25)$ and double-response conditions $(2.96 \mathrm{~s}$, $S D=1.22, p<.001, d=1.74)$. More information was sampled in the emotive message condition $(4.76 \mathrm{~s}, S D=1.33)$ compared to the double-response condition $(p=.004$, $d=1.41$ ). All other pairwise comparisons showed a nonstatistically significant resulting at the $p<.05$ level. All mean scores and standard deviations relating to the IST can be found in Table 3 .

\section{7-item Monetary Choice Questionnaire}

K-value. Data of one participant assigned to the doubleresponse condition were excluded from analysis due to an overall response consistency of $59 \%$ on the MCQ, indicative of potential random response choices. This figure of $59 \%$ is adequately lower than the $75 \%$ response consistency suggested by Kaplan, Lemley, Reed, and Jarmolowicz (2014) for meaningful analysis and therefore the data were removed, leaving 69 participants for this section of data analysis. Of note, three other participants, all in separate conditions, had an overall response consistency score of $70.37 \%$. While this falls marginally below the recommended $75 \%$ for inclusion, given the relatively modest number of participants in each group and the fact that these participants only fell marginally below the recommended threshold of consistency for one subcategory of delay period (i.e., large, medium, or short delay period), their data were retained for analysis.

The $k$-value is an estimate of discounting rate demonstrated by the participant, and the possible $k$-values, as assessed by the 27-item MCQ, ranging from 0.00016 and 0.25 , where a larger value represents a steeper discounting rate indicative of greater levels of choice impulsivity. A one-way betweenparticipants ANOVA showed that the difference in overall mean $k$-values across conditions reached statistical significance $\left[F(4,68)=7.30, p<.001, \eta^{2}=0.31\right]$. Bonferroni pairwise comparisons showed that relative to the control condition $(0.137, S D=0.085)$, only the emotive message condition $(0.027 \mathrm{~s}, S D=0.041, p=.003, d=1.65)$ and structure change condition $(0.052 \mathrm{~s}, S D=0.086, p=.043, d=$ $0.99)$ had $k$-values that differed to a statistically significant degree, where $k$-values were significantly lower indicating greater tolerance for reward delay. The highest overall $k$-value was found in the double-response condition $(0.155, S D=0.081)$, which was statistically significantly higher than both the emotive message $(p<.001, b=1.99)$ and structure change conditions ( $p=.007, b=1.23)$, as well as the informative message condition $(0.068, S D=0.076$, $p=.039, b=1.11)$, but was not statistically significantly higher when compared to the control condition. All other pairwise comparisons of means failed to reach statistical significance. Table 3 summarizes performances on the choice impulsivity tasks across the experimental conditions.

\section{DISCUSSION}

In support of the empirical findings from the second experiment, only the structure change condition resulted in improved response inhibition performance relative to a non-intervention control condition. In support of $\mathrm{H} 1$, participants in the structure change condition on average also demonstrated the highest $p$-correct values on the IST, where they also sampled more information before making a decision and demonstrated more deliberation over their decision, as demonstrated by longer choice latencies. Participants in the structure change condition also had a statistically significantly lower $k$-value on the MCQ when compared to the control condition, indicating a greater tolerance for larger delayed reward. The data therefore suggest that inducing motor cautiousness during EGM gambling has beneficial transfer effects that also reduce impulsive choice tendencies.

The fastest reaction times during gambling were found in the double-response condition, where reaction times were statistically significantly shorter when compared to all other conditions including the control condition. The worst performance on the motor response inhibition task, IST (in terms of lowest mean $p$-correct value), as well as highest $k$-value on the MCQ, was also found in the double-response condition, indicating that inducing rapid and impulsive motor responses is associated with impaired cognitive choice. However, differences in the percentage of successfully inhibited motor responses, $p$-correct scores, and $k$-values between the double-response and control condition just failed to reach statistical significance, meaning full support for $\mathrm{H} 2$ was not obtained.

Table 3. Mean (and standard deviation) of IST and MCQ values across experimental conditions

\begin{tabular}{lccrr}
\hline & \multicolumn{4}{c}{ Dependent variable } \\
\cline { 2 - 5 } Condition & IST choice latency (s) & IST balls sampled (1-19) & IST $p$-correct & MCQ $k$-value \\
\hline Control & $5.93(2.65)$ & $3.51(1.21)$ & $.82(.03)$ & $0.14(0.09)$ \\
Structure change & $9.19(2.78)$ & $5.14(1.28)$ & $.86(.03)$ & $0.05(0.09)$ \\
Emotive message & $9.76(3.22)$ & $4.76(1.33)$ & $.85(.04)$ & $0.03(0.04)$ \\
Informative message & $6.84(2.83)$ & $3.51(1.32)$ & $.82(.03)$ & $0.07(0.08)$ \\
Double-response & $5.19(2.28)$ & $2.96(1.22)$ & $.81(.04)$ & $0.15(0.08)$ \\
\hline
\end{tabular}

Note. Group comparisons for all variables reached statistical significance $(p<.001)$. Statistically significant Bonferroni comparisons: IST choice latency - STRU, EMOT $>$ CONT, DOUB. STRU $>$ INFO. IST balls sampled - STRU $>$ CONT, INFO, DOUB. EMOT $>$ DOUB. IST $p$-correct - STRU, EMOT > CONT, DOUB. STRUC > INFO. MCQ $k$-value - STRU, EMOT <CONT, DOUB. DOUB $>$ EMOT, INFO. CONT: control; STRU: structure change; EMOT: emotive message; INFO: informative message; DOUB: double-response; IST: Information Sampling Task; MCQ: Monetary Choice Questionnaire. 
Notably, IST $p$-correct scores were also statistically significantly higher in the emotive message condition compared to the control condition, as well as $k$-values on the MCQ in the emotive message condition being statistically significantly lower when compared to the control condition. Given the fact that the emotive message intervention failed to facilitate motor response inhibition means that increased motor cautiousness, while beneficial for cognitive choice in this gambling simulation, is not an essential factor in improving information sampling and delay discounting performance during gambling. Therefore, only partial support for H3 was obtained. This is because while structure change approaches that reduced impulsive action also reduced impulsive choice, the emotive message condition failed to reduce impulsive action and yet was able to reduce impulsive choice. Importantly, these group level differences in motor and cognitive performances cannot be attributed to baseline levels of trait impulsivity, given the lack of statistically significant differences in participant BIS-11 scores between experimental groups.

The findings of this study support existing evidence from neuroscience (Knoch et al., 2006), neuroimaging (Bunge et al., 2002; Wager et al., 2005), and behavioral approaches (Muraven \& Baumesiter, 2000; Verbruggen et al., 2012) that demonstrate significant overlaps between impulsive action and impulsive choice. Although the relationship between these constructs has received less attention in gambling, existing work exploring this relationship within a gambling context found that forcing gamblers to exercise greater caution over motor responses resulted in a preference for smaller and lower risk wagers in a novel gambling task (Verbruggen et al., 2012). This study extends these findings to a more realistic gambling activity and demonstrates that inducing motor cautiousness within slot machine gambling reduces impulsive choice tendencies. To the authors' knowledge, this is also the first empirical study to directly assess the efficacy of gambling harm-minimization tools in inducing motor cautiousness, and the impact this has on reflection impulsivity and delay discounting.

\section{Evidence for direct and indirect influence on impulsive choice}

The findings of this study suggest that a gambler's level of impulsive choice during gambling can be positively influenced via multiple processes. There is evidence for an "indirect" route, in which decision-making is shaped via a motor cautiousness transfer effect. There is also evidence that decision-making during gambling is influenced via a more "direct" route, whereby the presentation of messages containing responsible gambling information cause a gambler to reflect more carefully upon their decisions. The efficacy of responsible gambling messages in this direct process appears dependent upon the type of information delivered through the pop-up message, because levels of impulsive choice were significantly reduced only when emotive content was presented to the participant.

\section{Indirect cautiousness transfer account}

Arousal is a non-cognitive factor that has been shown to be influenced by the presence of cues requiring the withholding of motor responses (e.g., Jennings, van der Molen, Brock, \& Somsen, 1992; van Boxtel, van der Molen, Jennings, \& Brunia, 2001). However, like the study carried about by Stevens et al. (2015), there was no evidence in this gambling simulation to suggest that the association between motor cautiousness and cognitive choice in the structure change condition was due to subjective changes in arousal, given the non-significant change in arousal across all experimental conditions. Subjective arousal ratings in this study thus appear to corroborate findings from biological assessment of arousal that also shows no relationship between arousal and impulsivity transfer effects (e.g., Stevens et al., 2015). There was also a lack of evidence to suggest that the association between increased motor cautiousness and cognitive choice was a result of the structure change condition reducing the level of dissociation experienced by participants, nor was there evidence to suggest the association was related to changes in emotional valence.

Several studies suggest an association between withholding/cancelling motor responses in Go/No-Go and Stop Signal paradigms with subsequent approach behaviors (Houben \& Jansen, 2011; Lawrence, Verbruggen, Morrison, Adams, \& Chambers, 2014; Veling, Aarts, \& Stroebe, 2013). Being forced to exercise caution over motor responses in the structure change condition may have stimulated aversive centers in the brain, which are postulated by Dickinson and Dearing (1979) to act antagonistically with appetitive or approach centers. These authors also argue that the perceived affective dimension of a stimulus determines subsequent approach or avoidance behavior. Consistent with this notion, but outside of a gambling context, Dickinson and Balleine (2002) described an experiment in which inducing aversive defensive eye-blinks suppressed appetite jaw movements in rabbits. Furthermore, with human participants, Jones et al. (2011) demonstrated that cautiousness in a motor inhibition task using neutral stimuli resulted in reduced alcohol consumption in a later alcohol taste test, and was argued to be the result of the presence of stop signals stimulating the aversive/avoidance system within the brain.

Therefore, exercising motor control appears to have carryover effects to impulsive choice tasks, where participants are primed toward a more cautious decision-making style, where risk is valued as less desirable. This translated in this study as a willingness for participants in the structure change condition to make decisions on the IST based on more information (i.e., more balls removed from the urn and thus less uncertainty in decision-making) and taking more time to arrive at a decision as to the majority ball color, as well as being more tolerant of reinforcement delays in the MCQ.

\section{Direct cautiousness transfer account}

Participants exposed to the emotive message intervention had $p$-correct scores in the IST and $k$-values in the MCQ comparable to participants in the structure change condition. Given the fact that response inhibition performance for participants in the emotive message condition showed no improvement relative to a control condition, this rules out a motor cautiousness transfer effect being responsible for the 
improved performance on the cognitive choice tasks in this condition. Pop-up messages have been argued to encourage responsible gambling behavior by increasing a gambler's self-awareness over their behavior and engage gamblers in self-appraisal of that behavior (Monaghan, 2008). This appeared to be effective post-gambling on subsequent cognitive choice tasks, but had no impact on response inhibition during the gambling simulation in this study and is consistent with results from the second experiment.

One potential explanation for this is that pop-ups become effective during periods of time that allow for deliberation and reflection to occur. The rapid speed of play of slot machine gambling may override the effect of the pop-up message resulting in a lack of improved response inhibition during the slot machine gambling. It was only following the end of the gambling simulation that the effects of pop-up message exposure were evident. Arguably, the subsequent cognitive choice tasks were an example of a situation that encouraged deliberation, and this was therefore a situation in which the cognitive effects of the pop-up message exposure had opportunity to influence decision-making. Only the message containing emotive content (i.e., a warning of the potential familial and financial consequences of a loss of control during gambling) was effective as a harmminimization approach. Non-emotive message content appeared to fail to influence decisions, emphasizing the notion that the type of content displayed in pop-up messages is vital for their effectiveness as a harm-minimization tool.

\section{Argument for shared mechanism of change}

The positive impact of the emotive pop-up message on decision-making has been referred to here as a "direct" cautiousness transfer effect. The impact of the emotive popup message intervention on cognitive choice appears independent of a motor cautiousness effect, and can be regarded as a direct impact on decision-making. However, the impact of the structure change condition on decision-making has been referred to as an "indirect" transfer effect, because changes in structure appear to impact motor response processes that subsequently and indirectly transfer to wider aspects of decision-making involved in the cognitive choice tasks. It may be the case that there is a shared mechanism underlying both the direct and indirect pathways postulated here. Both the need to exercise greater caution over motor responses in the structure change condition, and the presentation of negative consequences associated with loss of control via the emotive pop-up message condition possibly reduce the hedonistic and motivational value of subsequent risk in favor of a more cautious and risk-averse approach. This potential explanation is consistent with Dickinson and Dearing's (1979) proposal of an antagonistic appetitive and aversive system within the brain.

\section{Caveats}

One of the limitations of this study is that the results can only be assumed to apply to healthy, non-problem gamblers. For example, problem gamblers have been shown to fail to properly activate inhibitory control networks within the brain and poor inhibition of prepotent responses has been associated with reduced capacity to remain abstinent following outpatient treatment for pathological gambling (Brevers et al., 2012). As a result, the effectiveness of implicating structural changes to gambling products to promote cautious motor responses remains unclear for problem gambling groups, which represents an important area of future research. One assumption made by the screening questions to check for current or previous periods of problem gambling is that participants had an absence of problem gambling symptomology. However, problem gambling severity lies along a continuum and therefore those participants who may be considered as low- or even moderate-risk problem gamblers may have gone undetected by the simple screening procedure, if the participant did not consider milder issues as problematic.

Furthermore, those with gambling-related issues may not have been aware that they had a problem. Use of a scale sensitive to these subcategories of problem gambling severity, such as the Problem Gambling Severity Index, could shed light on how these subgroups are influenced by the harm-minimization approaches investigated in this study. However, it is not the intention of this study for these successful harm-minimization approaches to help cure problem gambling in isolation. Instead, the intention is to help the much larger majority of gamblers who gamble without problems to remain in control during gambling and minimize the potential for harm caused by specific structural characteristics of gambling products (Harris \& Griffiths, 2018). However, this does not rule out the potential for future research to investigate how approaches encouraging motor cautiousness could have potential clinical utility alongside other treatment approaches for problem gamblers.

Although the participants were screened for problem gambling behavior, there is a range of potential behavioral and clinical factors not screened that may drive the group-level differences in impulsivity found in the betweenparticipant design. Although baseline levels of impulsivity were controlled across groups according to BIS-11 scores (which include measures of secondary factors including motor impulsivity), participants were not matched on response inhibition according to performance on impulsivity task performance outside of a gambling context. In addition, data pertaining to potential clinical problems were not obtained from participants. For example, ADHD, other impulse and compulsive control disorders, as well as other addictions represent underlying issues that could impact group-level differences for motor and choice impulsivity measures found in this study, particularly given the relatively small number of participants $(n=14)$ in each of the five conditions.

Although to the authors' knowledge, this is the first study to assess impulsivity transfer effects using widely used cognitive measures within a gambling simulation, it is not clearcut as to how decisions in the cognitive choice tasks translate to real-world gambling-related decisions. It would be difficult to argue against the notion that making probabilistic decisions based on more information and increased deliberation time have advantages within a wide range of disciplines, including gambling. In addition, preferences for larger delayed rewards over immediate but smaller reward are advantageous from a utility perspective. What is required is follow-up research assessing how these positive decision-making features relate 
to within- and between-gambling session factors, including loss-chasing behavior, monetary spend during gambling, time spent gambling, and gambling frequency.

\section{CONCLUSIONS}

This experimental investigation using regular non-problem gamblers demonstrated that structural modifications to slot machine gambling can impact executive control domains, including motor response inhibition and delay discounting, as well as information sampling. These effects were found to be independent of trait impulsivity. There was also evidence that inducing motor cautiousness by forcing gamblers to discriminate between motor responses had positive indirect transfer effects to wider aspects of cognitive choice, suggesting impulsive choice and impulsive action have related underlying processes. The use of emotive content in pop-up messages directly facilitated decision-making in cognitive choice tasks, although this effect was independent of a motor cautiousness transfer account. Furthermore, these direct and indirect effects appeared independent of subjective changes in arousal, dissociation, and valence. Inducing motor cautiousness during gambling appears to have global benefits for selfcontrol and has the potential to assist healthy gamblers avoid behaviors that may lead to risky gambling practices. Consequently, future research should assess how inducing motor cautiousness transfers to specific gambling factors such as time and monetary spend, and loss chasing behaviors, as well as the impact of inducing motor cautiousness during slot machine gambling on gamblers with varying degrees of problem gambling severity.

Funding sources: AH and MDG have received funding for research projects in the area of gambling education for young people, social responsibility in gambling and gambling treatment from GambleAware (formerly the Responsibility in Gambling Trust), a charitable body that funds its research program based on donations from the gambling industry.

Authors' contribution: AH collected and analyzed the data. AH and MDG designed the study and initially interpreted the data. AH wrote the first draft of the paper, which then underwent a number of iterations with MDG who supervised the study. DK contributed to the final draft of the paper. All authors have contributed, revised, and agreed with the final submitted manuscript.

Conflict of interest: DK reports no financial or other relationship relevant to the subject of this paper. MDG's university currently receives funding from Norsk Tipping (the gambling operator owned by the Norwegian Government) for gambling research. He has received funding for a number of research projects in the area of gambling education for young people, social responsibility in gambling, and gambling treatment from GambleAware (formerly the Responsibility in Gambling Trust), a charitable body that funds its research program based on donations from the gambling industry. He also undertakes consultancy for various gaming companies in the area of social responsibility in gambling.

\section{REFERENCES}

Anokhin, A. P., Golosheykin, S., \& Mulligan, R. C. (2015). Longterm test-retest reliability of delayed reward discounting in adolescents. Behavioural Processes, 111, 55-59. doi:10.1016/ j.beproc.2014.11.008

Arce, E., \& Santisteban, C. (2006). Impulsivity: A review. Psicothema, 18(2), 213-220.

Ávila, C., Cuenca, I., Félix, V., Parcet, M. A., \& Miranda, A. (2004). Measuring impulsivity in school-aged boys and examining its relationship with ADHD and ODD ratings. Journal of Abnormal Child Psychology, 32(3), 295-304. doi:10.1023/B: JACP.0000026143.70832.4b

Bechara, A. (2002). The neurology of social cognition. Brain, 125(8), 1673-1675. doi:10.1093/brain/awf169

Bechara, A., Damasio, H., \& Damasio, A. R. (2000). Emotion, decision making and the orbitofrontal cortex. Cerebral Cortex, 10(3), 295-307. doi:10.1093/cercor/10.3.295

Bechara, A., Damasio, H., Damasio, A. R., \& Anderson, S. W. (1994). Insensitivity to future consequences following damage to human prefrontal cortex. Cognition, 50(1-3), 7-15.

Bickel, W. K., Jarmolowicz, D. P., Mueller, E. T., Gatchalian, K. M., \& McClure, S. M. (2012). Are executive function and impulsivity antipodes? A conceptual reconstruction with special reference to addiction. Psychopharmacology, 221(3), 361-387. doi:10.1007/s00213-012-2689-x

Blaszczynski, A., Cowley, E., Athony, C., \& Kinsley, K. (2016). Breaks in play: Do they achieve intended aims? Journal of Gambling Studies, 32(2), 789-800. doi:10.1007/s10899-0159565-7

Bogacz, R., Wagenmakers, E. J., Forstmann, B. U., \& Nieuwenhuis, S. (2010). The neural basis of the speed-accuracy tradeoff. Trends in Neurosciences, 33(1), 10-16. doi:10.1016/j.tins.2009. 09.002

Bradly, M. M. (1994). Emotional memory: A dimensional analysis. In S. van Goozen, N. E.van de Poll, \& J. A. Sergeant (Eds.), The emotions: Essays on emotion theory (pp. 97-134). Hillsdale, NJ: Lawrence Erlbaum Associates.

Bradly, M. M., \& Lang, P. J. (1994). Measuring emotion: The selfassessment manikin and the semantic differential. Journal of Behaviour, Therapy, and Experimental Psychiatry, 25(1), 49-59. doi:10.1016/0005-7916(94)90063-9

Brevers, D., Cleeremans, A., Verbruggen, F., Bechara, A., Kornreich, C., Verbanck, P., \& Noel, X. (2012). Impulsive action but not impulsive choice determines problem gambling severity. PLoS One, 7(11), e50647. doi:10.1371/ journal.pone.0050647

Broos, N., Diergaarde, L., Schoffelmeer, A. N., Pattij, T., \& De Vries, T. J. (2012). Trait impulsive choice predicts resistance to extinction and propensity to relapse to cocaine seeking: A bidirectional investigation. Neuropsychopharmacology, 37(6), 1377-1386. doi:10.1038/npp.2011.323

Broos, N., Schmaal, L., Wiskerke, J., Kostelijk, L., Lam, T., Stoop, N., Weierink, L., Ham, J., de Geus, E. J. C., Schoffelmeer, A. N. M., van den Brink, W., Veltman, D. J., de Vries, T. J., 
Pattij, T., \& Goudriaan, A. E. (2012). The relationship between impulsive choice and impulsive action: A cross-species translational study. PLoS One, 7(5), e36781. doi:10.1371/journal. pone. 0036781

Bunge, S. A., Dudukovic, N. M., Thomason, M. E., Vaidya, C. J., \& Gabrieli, J. D. (2002). Immature frontal lobe contributions to cognitive control in children: Evidence from fMRI. Neuron, 33(2), 301-311. doi:10.1016/S0896-6273(01) 00583-9

Chambers, C. D., Bellgrove, M. A., Stokes, M. G., Henderson, T. R., Garavan, H., Robertson, I. H., Morris, A. P., \& Mattingley, J. B. (2006). Executive "brake failure" following deactivation of human frontal lobe. Journal of Cognitive Neuroscience, 18(3), 444-455. doi:10.1162/089892906775990606

Chambers, C. D., Garavan, H., \& Bellgrove, M. A. (2009). Insights into the neural basis of response inhibition from cognitive and clinical neuroscience. Neuroscience and Biobehavioral Reviews, 33(5), 631-646. doi:10.1016/j.neubiorev. 2008.08.016

Clark, L., Lawrence, A. J., Astley-Jones, F., \& Gray, N. (2009). Gambling near-misses enhance motivation to gamble and recruit win-related brain circuitry. Neuron, 61(3), 481-490. doi:10.1016/j.neuron.2008.12.031

Clark, L., Robbins, T. W., Ersche, K. D., \& Sahakian, B. J. (2006). Reflection impulsivity in current and former substance users. Biological Psychiatry, 60(5), 515-522. doi:10.1016/j.biopsych. 2005.11.007

Cohen, J. R., \& Lieberman, M. D. (2010). The common neural basis of exerting self-control in multiple domains. In R. R. Hassin, K. N. Ochsner, \& Y. Trope (Eds.), Oxford series in social cognition and social neuroscience. Self-control in society, mind, and brain (pp. 141-160). Oxford, UK: Oxford University Press.

Delfabbro, P. H. (2006). Dissociation and problem gambling: A critical overview. In C. Allcock (Ed.), Current issues related to dissociation (pp. 10-14). Melbourne, Australia: Australian Gaming Council.

Dickinson, A., \& Balleine, B. (2002). The role of learning in the operation of motivational systems. In H. Pashler \& R. Gallistel (Eds.), Steven's handbook of experimental psychology: Learning, motivation, and emotion (pp. 497-533). Chichester, UK: Wiley.

Dickinson, A., \& Dearing, M. F. (1979). Appetitive-aversive interactions and inhibitory processes. In A. Dickinson \& R. A. Boakes (Eds.), Mechanisms of learning and motivation (pp. 203-231). Hillsdale, NJ: Lawrence Erlbaum Associates.

Diergaarde, L., Pattij, T., Poortvliet, I., Hogenboom, F., De Vries, W., Schoffelmeer, A. N., \& De Vries, T. J. (2008). Impulsive choice and impulsive action predict vulnerability to distinct stages of nicotine seeking in rats. Biological Psychiatry, 63(3), 301-308. doi:10.1016/j.biopsych.2007.07.011

Duncan, J. (1986). Disorganisation of behaviour after frontal lobe damage. Cognitive Neuropsychology, 3(3), 271-290. doi:10. 1080/02643298608253360

Gupta, R., \& Derevensky, J. (1998). Adolescent gambling behavior: A prevalence study and examination of the correlates associated with excessive gambling. Journal of Gambling Studies, 14(4), 319-345. doi:10.1023/A:1023068 925328

Harris, A., \& Griffiths, M. D. (2018). The impact of speed of play in gambling on psychological and behavioural factors: A critical review. Journal of Gambling Studies, 34(2), 393-412. doi:10.1007/s10899-017-9701-7

Holmes, J., Hever, T., Hewitt, L., Ball, C., Taylor, E., Rubia, K., \& Thapar, A. (2002). A pilot twin study of psychological measures of attention deficit hyperactivity disorder. Behavioral Genetics, 32(6), 389-395. doi:10.1023/A:1020846 426166

Houben, K., \& Jansen, A. (2011). Training inhibitory control: A recipe for resisting sweet temptations. Appetite, 56(2), 345-349. doi:10.1016/j.appet.2010.12.017

Jennings, J. R., van der Molen, M. W., Brock, K., \& Somsen, R. J. (1992). On the synchrony of stopping motor responses and delaying heartbeats. Journal of Experimental Psychology, 18(2), 422-436. doi:10.1037//0096-1523.18.2.422

Jones, A., Guerrieri, R., Fernie, G., Cole, J., Goudie, A., \& Field, M. (2011). The effects of priming restrained versus disinhibited behaviour on alcohol-seeking in social drinkers. Drug and Alcohol Dependence, 113(1), 55-61. doi:10.1016/j.drugalcdep. 2010.07.006

Kaplan, B. A., Lemley, S. M., Reed, D. D., \& Jarmolowicz, D. P. (2014). 21- and 27-Item Monetary Choice Questionnaire Automated Scorer (spreadsheet application). Retrieved March 28, 2018, from http://hdl.handle.net/1808/15424

Kirby, K. N., Petry, N. M., \& Bickel, W. K. (1999). Heroin addicts have higher discount rates for delayed rewards than nondrug-using controls. Journal of Experimental Psychology, 128(1), 78-87. doi:10.1037/0096-3445.128.1.78

Knoch, D., Gianotti, L. R. R., Pascual-Leone, A., Treyer, V., Regard, M., Hohmann, M., \& Brugger, P. (2006). Disruption of right prefrontal cortex by low-frequency repetitive transcranial magnetic stimulation induces risk-taking behavior. Journal of Neuroscience, 26(24), 6469-6472. doi:10.1523/ JNEUROSCI.0804-06.2006

Kocka, A., \& Gagnon, J. (2014). Definition of impulsivity and related terms following traumatic brain injury: A review of the different concepts and measures used to assess impulsivity, disinhibition, and other related concepts. Behavioral Sciences, 4(4), 352-370. doi:10.3390/bs4040352

Konishi, S., Nakajima, K., Uchida, I., Kikyo, H., Kameyama, M., \& Miyashita, Y. (1999). Common inhibitory mechanism in human inferior prefrontal cortex revealed by event related functional MRI. Brain, 122(5), 981-991. doi:10.1093/brain/ 122.5.981

Kuley, N. B., \& Jacobs, D. F. (1988). The relationship between dissociative-like experiences and sensation seeking among social and problem gamblers. Journal of Gambling Studies, 4(3), 197-207. doi:10.1007/BF01018332

Lang, P. J. (1980). Behavioral treatment and bio-behavioral assessment: Computer applications. In J. B. Sidowski, J. H. Johnson, \& T. A. Williams (Eds.), Technology in mental health care delivery systems (pp. 119-137). Norwood, NJ: Ablex.

Lang, P. J., Greenwald, M. K., Bradley, M. M., \& Hamm, A. O. (1993). Looking at pictures: Evaluative, facial, visceral, and behavioral responses. Psychophysiology, 30(3), 261-273. doi:10.1111/j.1469-8986.1993.tb03352.x

Langley, K., Marshall, L., van den Bree, M., Thomas, H., Owen, M., O'Donovan, M., \& Thapar, A. (2004). Association of the dopamine D4 receptor gene 7-repeat allele with neuropsychological test performance of children with ADHD. American Journal of Psychiatry, 161(1), 133-138. doi:10.1176/appi. ajp.161.1.133 
Lawrence, N. S., Verbruggen, F., Morrison, S., Adams, R. C., \& Chambers, C. D. (2014). Stopping to food can reduce intake: Effects of stimulus-specificity and individual differences in dietary restraint. Appetite, 85, 91-103. doi:10.1016/j.appet.2014.11.006

Matta, A. D., Gonçalves, F., \& Bizarro, L. (2012). Delay discounting: Concepts and measures. Psychology \& Neuroscience, 5(2), 135-146. doi:10.3922/j.psns.2012.2.03

Matthews, S. C., Simmons, A. N., Arce, E., \& Paulus, M. P. (2005). Dissociation of inhibition from error processing using a parametric inhibitory task during functional magnetic resonance imaging. Neuroreport, 16(7), 755-760.

Monaghan, S. (2008). Review of pop-up messages on electronic gaming machines as a proposed responsible gambling strategy. International Journal of Mental Health and Addiction, 6(2), 214-222. doi:10.1007/s11469-007-9133-1

Muraven, M., \& Baumeister, R. F. (2000). Self-regulation and depletion of limited resources: Does self-control resemble a muscle? Psychological Bulletin, 126(2), 247-259. doi:10. 1037/0033-2909.126.2.247

Parke, A., Harris, A., Parke, J., \& Goddard, P. (2015). Understanding within-session loss-chasing: An experimental investigation of the impact of stake size on cognitive control. Journal of Gambling Studies, 32(2), 721-735. doi:10.1007/ s10899-015-9570-x

Patton, J. H., Stanford, M. S., \& Barratt, E. S. (1995). Factor structure of the Barratt Impulsiveness Scale. Journal of Clinical Psychology, 51(6), 768-774. doi:10.1002/1097-4679 (199511)51:6<768::AID-JCLP2270510607>3.0.CO;2-1

Peirce, J. W. (2007). PsychoPy - Psychophysics software in Python. Journal of Neuroscience Methods, 162(1-2), 8-13. doi:10.1016/j.jneumeth.2006.11.017

Peters, J., \& Buchel, C. (2011). The neural mechanisms of intertemporal decision-making: Understanding variability. Trends in Cognitive Science, 15(5), 227-239. doi:10.1016/j.tics. 2011.03.002

Rosello, J., Munar, C., Justo, S., \& Arias, R. (1998). Effects of alcohol on divided attention and on accuracy of attentional shift. Psicothema (Oviedo), 10, 65-73.

Shallice, T. (1982). Specific impairments of planning. Philosophical Transactions of the Royal Society of London, Series B, Biological Sciences, 298(1089), 199-209. doi:10.1098/rstb. 1982.0082
Solanto, M. V., Abikoff, H., Sonuga-Barke, E., Schachar, R., Logan, G. D., Wigal, T., Hechtman, L., Hinshaw, S., \& Turkel, E. (2001). The ecological validity of delay aversion and response inhibition as measures of impulsivity in $\mathrm{AD} / \mathrm{HD}$ : A supplement to the NIMH multimodal treatment study of AD/HD. Journal of Abnormal Child Psychology, 29(3), 215-228. doi:10.1023/A:1010329714819

Spinella, M. (2004). Neurobehavioral correlates of impulsivity: Evidence of prefrontal involvement. International Journal of Neuroscience, 114(1), 95-104. doi:10.1080/002074504902 49347

Stevens, T., Brevers, D., Chambers, C. D., Lavric, A., McLaren, I. P. L., Mertens, M., Noël, X., \& Verbruggen, F. (2015). How does response inhibition influence decision-making when gambling? Journal of Experimental Psychology: Applied, 21(1), 15-36. doi:10.1037/xap0000039

van Boxtel, G. J., van der Molen, M. W., Jennings, J. R., \& Brunia, C. H. (2001). A psychophysiological analysis of inhibitory motor control in the stop-signal paradigm. Biological Psychology, 58(3), 229-262. doi:10.1016/S03010511(01)00117-X

van den Bos, G. R. (2007). APA dictionary of psychology. Washington, DC: American Psychological Association.

Veling, H., Aarts, H., \& Stroebe, W. (2013). Stop signals decrease choices for palatable foods through decreased food evaluation. Frontiers in Psychology, 4, 875. doi:10.3389/ fpsyg.2013.00875

Verbruggen, F., Adams, R., \& Chambers, C. D. (2012). Proactive motor control reduces monetary risk taking in gambling. Psychological Science, 23(7), 805-815. doi:10.1177/ 0956797611434538

Verbruggen, F., Aron, A. R., Stevens, M. A., \& Chambers, C. D. (2010). Theta burst stimulation dissociates attention and action updating in human inferior frontal cortex. Proceedings of the National Academy of Sciences of the United States of America, 107(31), 13966-13971. doi:10.1073/ pnas. 1001957107

Wager, T. D., Sylvester, C. Y., Lacey, S. C., Nee, D. E., Franklin, M., \& Jonides, J. (2005). Common and unique components of response inhibition revealed by fMRI. Neuroimage, 27(2), 323-340. doi:10.1016/j.neuroimage. 2005.01 .054 